\title{
Insulin resistance in relation to clinical, endocrine and metabolic profile of infertile women with polycystic ovary syndrome
}

\author{
Shakeela Ishrat, Marufa Hossain and Subrata Kumar Biswas
}

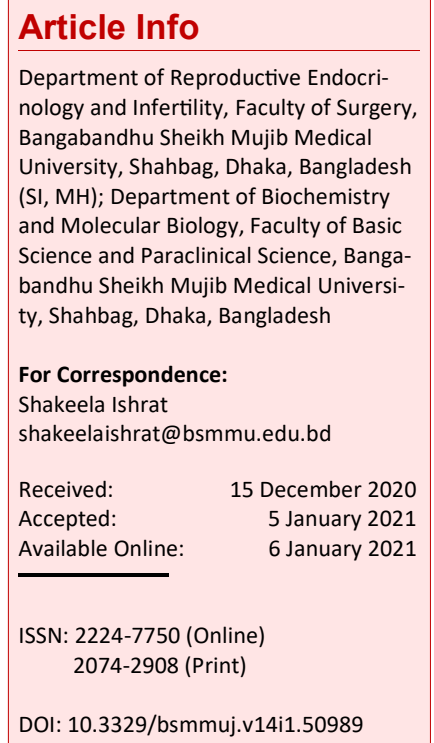

Cite this article:

Ishrat $S$, Hossain M, Biswas SK. Insulin resistance in relation to clinical, endocrine and metabolic profile of infertile women with polycystic ovary syndrome. Bangabandhu Sheikh Mujib Med Univ J. 2021; 14: 1-6.

\section{Copyright:}

The copyright of this article is retained by the author(s) [Atribution CC-By 4.0]

\section{Available at:}

www.banglajol.info

A Journal of Bangabandhu Sheikh Mujib Medical University, Dhaka, Bangladesh

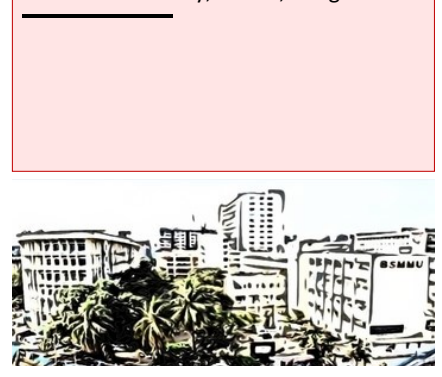

\begin{abstract}
The objective of this study is to explore how hyperinsulinemia and insulin resistance relate to the clinical, endocrine and metabolic factors in the infertile women with polycystic ovary syndrome. This study was conducted on 121 consecutive infertile women with polycystic ovary syndrome attending the Infertility unit from January 2017 to December 2017. They were divided into two groups: insulin resistant and insulin sensitive. There was significant difference in body mass index and waist circumference between the two groups. Serum lipids were not associated with insulin resistance. Hyperinsulinemia was significantly associated with metabolic syndrome. Reducing body mass index and waist circumference may improve insulin resistance in infertile women with polycystic ovary syndrome. Screening the infertile women with polycystic ovary syndrome for hyperinsulinemia and insulin resistance and subsequent counseling is recommended to address the long-term risks of metabolic syndrome.
\end{abstract}

\section{Introduction}

Infertility in a couple is failure to achieve pregnancy within one year of conjugal life. Polycystic ovary syndrome is a common cause of infertility in young women. It is a chronic endocrine and metabolic disorder characterized by hyperandrogenism and anovulation. Diagnostic criteria of polycystic ovary syndrome are any two of the three features present in a woman: anovulation or oligo-ovulation mostly evident as menstrual irregularities, hyperandrogenism manifested as hirsutism and/or acne, and sonographic appearance of enlarged ovaries with central echogenic area and peripheral multiple follicles.1 Insulin resistance or subnormal activity of insulin in glucose metabolism is a common feature in obese, and to a lesser extent in lean polycystic ovary syndrome women.2 Insulin resistance associated with hyperinsulinemia is prevalent in approximately half of the women with polycystic ovary syndrome. The factors leading to this condition includes resistance of peripheral tissues to insulin and reduced hepatic degradation of insulin. 3 Apart from risking impaired glucose tolerance, increased circulating insulin levels stimulate increased ovarian androgen production. There is elevated pulse frequency and amplitude of luteinizing hormone secretion in polycystic ovary syndrome. The action of luteinizing hormone is aggravated by insulin. The luteinizing hormone and insulin together sti- mulates ovarian androgen production. Intra ovarian androgen excess inhibits follicular maturation and ovulation in infertile women with polycystic ovary syndrome. These insulin actions on ovaries are enhanced at the same time when insulin action on glucose metabolism is inhibited in insulin resistant women with polycystic ovary syndrome. $\underline{4-7}$ The basic endocrine changes in infertile women with polycystic ovary syndrome is hyperandrogenism causing anovulation which in turn is aggravated by hyperinsulinemia and insulin resistance.

Measurement of fasting insulin is an easy marker of insulin resistance. The fasting glucose to insulin ratio or Homeostatic Model Assessment of Insulin resistance (HOMA-IR) is another sensitive and specific marker. 3 Euglycemic hyperinsulinemic clamp is the gold standard method for measuring insulin resistance or insulin sensitivity. The method is expensive and time consuming, with the need of two intravenous lines and frequent bedside blood glucose measurements. HOMA-IR is based on single measurements of fasting insulin and fasting blood glucose, derived from the formula fasting insulin $(\mu \mathrm{U} / \mathrm{mL})$ multiplied by fasting glucose $(\mathrm{mmol} / \mathrm{L})$, divided by 22.5. HOMA-IR levels cut off $\geq 3.8$ is used for insulin resistance. $\underline{8}$ For South Asian population, the cut off value is lower.- $\mathrm{HOMA}$-IR method is more applicable to clinical and ambulatory settings. 
Insulin resistance is believed to be associated with clinical, endocrine and metabolic factors in women with polycystic ovary syndrome. Insulin resistance is more in polycystic ovary syndrome women with oligomenorrhea.9 Other clinical features such as body mass index, waist circumference, hirsutism and acanthosis nigricans (a brown or black velvety pigmentation usually on the back of neck) have been associated with insulin resistance. $\frac{8,10}{10}$ Among the endocrine factors, free testosterone increases with insulin resistance. 11 Dysglycemia, dyslipidemia and metabolic syndrome are the metabolic factors linked to insulin resistance. $\underline{\text { 12-14 }}$

There is no apparent change in insulin resistance in about $50 \%$ women with polycystic ovary syndrome. 3 Therefore insulin resistance and hyperinsulinemia may not be the primary factor in all women with polycystic ovary syndrome. Infertile women with polycystic ovary syndrome are assumed to present a selected population with more severe ovulation dysfunction. Insulin sensitizers like metformin reduces insulin resistance and causes spontaneous ovulation and pregnancy in some women. Clinical trials have confirmed effectiveness of metformin in the treatment of polycystic ovary syndrome related infertility. -3 However, studies regarding relevance of insulin resistance to other parameters are few in the selected population of infertile women with polycystic ovary syndrome.

The objective of this study is to explore how hyperinsulinemia and insulin resistance relate to the clinical, endocrine and metabolic factors in the infertile women with polycystic ovary syndrome.

\section{Materials and Methods}

This was a cross-sectional study of 121 infertile women with polycystic ovary syndrome who attended the Infertility unit of the Department of Obstetrics and Gynecology at Bangabandhu Sheikh Mujib Medical University from January 2017 to December 2017. The women attended sequentially, consented for study participation and were recruited at first visit. They were not exposed to metformin or the lifestyle modification for the last three months. Being infertile women, they were not exposed to oral contraceptive pills for cycle regulation or hirsutism. Consent was taken regarding participation in the study. Data collection was accomplished by maintaining adequate privacy and confidentiality and without any physical harm abiding by Helsinki declaration.

The women were evaluated by clinical history, examination, TVS (transvaginal sonogram) and fasting blood samples for endocrine and metabolic parameters. Eligibility criteria were infertile women with age range 15-40 years and any two of the following: i) infrequent menstruation (cycle length
$>35$ days), ii) hirsutism with modified Ferriman Galway score $\geq 8$, iii) at least one enlarged $\left(>10 \mathrm{~cm}^{3}\right)$ polycystic ovary at TVS. 1 The women were divided into two groups according to their HOMA-IR (Homeostatic Model Assessment of Insulin Resistance), estimated from fasting glucose and insulin levels. Those with HOMA-IR at or more than 2 were Group I (insulin resistant) and those with levels less than 2 in Group II (insulin sensitive).

Anthropometric measurements

\section{Body mass index}

Weight was measured on a beam scale to within 0.1 $\mathrm{kg}$ in light clothing without shoes. Subjects stood straight with both feet in firm contact with the surface, looking ahead with hands not touching any surface. Heights were measured to $0.1 \mathrm{~cm}$ using a wall mounted stadiometer. Body mass index was calculated as weight in kilogram divided by the square of height in meters.

\section{Waist circumference}

Waist circumference was measured to the nearest $0.1 \mathrm{~cm}$ with a non-stretchable measuring tape. The subject stood straight. The tape was placed, at the end of exhalation, horizontally between the last floating rib or lower costal margin and the iliac crest. Hip circumference was measured to the nearest $0.1 \mathrm{~cm}$ at the level of pubic symphysis and the point of greatest posterior extension of the buttocks. The waist circumference was divided by hip circumference to give waist hip ratio.

\section{Biochemical assay}

All the women had hormone analysis and oral glucose tolerance test. Fasting blood samples were drawn in follicular phase cycle day 2 to day 5. This was to avoid any possible effects of sex steroids on insulin action. Venous blood samples were drawn after an overnight fast of at least 8 hours. Fasting blood glucose was measured by hexokinase method (CI4100 ARCHITECT USA). All hormones were measured by Chemiluminiscent Microparticle Immunoassay (CI 4100 ARCHITECT USA)

\section{Cut-off values}

The cut-off value of waist circumference of Asian women for defining central obesity is $\geq 80 \mathrm{~cm}$ (International Diabetes Federation). $\underline{14}$ Hyper-androgenemia was defined when serum total testosterone was $>60 \mathrm{ng} / \mathrm{mL}$. High LH:FSH ratio was $>1$. Hyperinsulinemia was $\geq 10 \mathrm{mIU} / \mathrm{L}$ and the insulin resistance was HOMA IR $\geq 2$. Fasting insulin was $9.25 \mu \mathrm{IU} / \mathrm{mL}$ at $75^{\text {th }}$ percentile of Pakistani population. HOMA-IR was 1.93 at the $75^{\text {th }}$ percentile of Indian population and 1.82 at the75th percentile of Pakistani population.? For the sake of simplicity we rounded up the cut-off value HOMA-IR to 2 and the cut-off value of fasting insulin to $10 \mathrm{mIU} / \mathrm{L}$. 
Table I

\section{Clinical, endocrine and metabolic parameters in infertile polycystic ovary syndrome}

\begin{tabular}{|c|c|c|c|}
\hline Parameters & $\begin{array}{r}\text { Insulin resistant } \\
(\mathrm{n}=42)\end{array}$ & $\begin{array}{r}\text { Insulin sensi- } \\
\text { tive }(\mathrm{n}=79)\end{array}$ & $\mathrm{p}$ value \\
\hline Age (years) & $25.9 \pm 3.9$ & $25.5 \pm 3.9$ & 0.649 \\
\hline BMI $\left(\mathrm{kg} / \mathrm{m}^{2}\right)$ & $27.5 \pm 3.1$ & $26.1 \pm 3.1$ & $<0.05$ \\
\hline $\mathrm{WC}(\mathrm{cm})$ & $92.5 \pm 10.3$ & $90.3 \pm 10.3$ & 0.244 \\
\hline FSH (IU/L) & $4.9 \pm 1.7$ & $5.4 \pm 1.8$ & 0.135 \\
\hline $\mathrm{LH}^{*}(\mathrm{IU} / \mathrm{L})$ & 5.4 & 7.0 & 0.098 \\
\hline LH: FSH* & 1.3 & 1.3 & 0.402 \\
\hline Total testosterone* $(\mathrm{ng} / \mathrm{dL})$ & 49.5 & 49.7 & 0.548 \\
\hline $\mathrm{TSH}(\mathrm{mIU} / \mathrm{L})$ & $2.8 \pm 1.3$ & $2.4 \pm 1.3$ & 0.189 \\
\hline Prolactin* (ng/dl) & 12.2 & 11.4 & 0.786 \\
\hline Fasting insulin* $(\mathrm{mIU} / \mathrm{L})$ & 19.0 & 11.8 & $<0.05$ \\
\hline HOMA-IR* & 2.5 & 1.10 & $<0.05$ \\
\hline Fasting blood sugar* $(\mathrm{mmol} / \mathrm{L})$ & 4.9 & 4.8 & 0.423 \\
\hline Blood sugar 2 hours* (mmol/L) & 6.1 & 6.2 & 0.326 \\
\hline Total cholesterol* $(\mathrm{mg} / \mathrm{dL})$ & 188 & 184.5 & 0.325 \\
\hline Triglyceride* $^{*}(\mathrm{mg} / \mathrm{dL})$ & 138 & 129 & 0.325 \\
\hline LDL-cholesterol $^{*}(\mathrm{mg} / \mathrm{dL})$ & 132 & 124 & 0.418 \\
\hline HDL-cholesterol (mg/dL) & $39.0 \pm 6.3$ & $38.8 \pm 7.1$ & 0.864 \\
\hline
\end{tabular}

\section{Statistical analysis}

Statistical analysis was done by SPSS (Statistical Package for Social Science) version 23. The anthropometric, endocrine and metabolic parameters were compared between the two groups by independent sample t-test for variables with normal distribution and non-parametric tests (Mann Whitney $U$ test and Kruskal Wallis test) for variables with non-Gaussian distribution. Statistical significance (two-tailed) was defined as $\mathrm{p} \leq 0.05$.

\section{Results}

Of the 121 infertile women with polycystic ovary syndrome, $42(34.7 \%)$ are insulin resistant (HOMAIR $\geq 2)$ and $79(65.3 \%)$ were insulin sensitive (HOMA -IR $<2$ ). Table I summarizes the clinical, endocrine and metabolic profile of the women in two groups, insulin resistant and insulin sensitive.

Independent sample t-test and independent sample Mann Whitney U test to compare the continuous variables between two groups found no significant differences in clinical, endocrine and metabolic para -meters except HOMA-IR, fasting insulin and body mass index.

Analysis by $2 \times 2$ contingency table and Chisquared and Fishers Exact test to see the association of hyperinsulinemia (fasting insulin $\geq 10 \mathrm{mIU} / \mathrm{L}$ ) with different characteristics revealed that there was significant association with metabolic syndrome only (Table II).

Kruskal Wallis test of analysis of variance was done between 4 groups of HOMA IR (Table III). There was significant difference in fasting insulin and fasting blood glucose of the groups which is selfexplanatory. In addition, there was statistically significant difference in body mass index and waist circumference of the groups.

\section{Table III}

$\begin{gathered}\text { HOMA IR groups according to quartiles in } \\
\text { infertile women with polycystic ovary } \\
\text { syndrome for analysis of variance }\end{gathered}$
\begin{tabular}{lll} 
Groups & HOMA-IR & $\mathrm{n}$ \\
\hline 1 & $\leq 0.85$ & 34 \\
2 & $0.85<, \leq 1.6$ & 31 \\
3 & $1.6<, \leq 2.3$ & 28 \\
4 & $>2.3$ & 28 \\
\hline
\end{tabular}

\section{Discussion}

The study explores the relation of insulin resistance

Table II

Association of hyperinsulinemia (fasting insulin $\geq 10 \mathrm{mIU} / \mathrm{L}$ ) with clinical, endocrine and metabolic characteristics

\begin{tabular}{|c|c|c|c|}
\hline Characteristics & $\begin{array}{c}\text { Women with } \\
\text { hyperinsulinemia }\end{array}$ & $\begin{array}{l}\text { Women without } \\
\text { hyperinsulinemia }\end{array}$ & $\mathrm{p}$ value \\
\hline Central obesity & $69.3 \%$ & $25.7 \%$ & 0.255 \\
\hline Dysglycemia (Fasting blood sugar $\geq 5.6 \mathrm{mmol} / \mathrm{L}$ ) & $76.7 \%$ & $16.7 \%$ & 0.120 \\
\hline High total cholesterol $(\mathrm{TC}>250 \mathrm{mg} / \mathrm{dL})$ & $78.0 \%$ & $19.5 \%$ & 0.117 \\
\hline Hypertriglyceridemia (TG >150 mg/dL) & $64.4 \%$ & $31.1 \%$ & 0.360 \\
\hline High Low density cholesterol (LDL >100/dL) & $72.9 \%$ & $22.9 \%$ & 0.442 \\
\hline Low High density cholesterol (HDL<50 mg/dL) & $67.6 \%$ & $28.4 \%$ & 0.144 \\
\hline Metabolic syndrome & $71.7 \%$ & $20.8 \%$ & $<0.05$ \\
\hline
\end{tabular}


to clinical, endocrine and metabolic parameters of infertile women with polycystic ovary syndrome. The prevalence of insulin resistance (HOMA-IR $\geq 2$ ) in our population of infertile women with polycystic ovary syndrome is $34.7 \%$. The association of insulin resistance with body mass index and waist circumference is statistically significant. Hyperinsulinemia is associated with the risk of metabolic syndrome. There is no significant difference in other clinical, endocrine and metabolic parameters between insulin sensitive and insulin resistant groups.

The variables are positively skewed in the two groups (insulin resistant and insulin sensitive) as we have a selected population of infertile women with polycystic ovary syndrome, likely to have more severe endocrine and metabolic dysfunction. Relevance of anthropometric indices to insulin resistance measured by HOMA IR (Homeostatic Model Assessment) may have ethno-cultural differences. South Asian people has been found to be insulin resistant at a lower threshold than their Caucasian counterpart matched for body mass index.11 Due to probable genetic or cultural factors, polycystic ovary syndrome women in South Asia have higher prevalence of central obesity and insulin resistance than other ethnic groups. $\underline{15}$ For a given waist circumference, glucose levels may be higher in South Asian women than Europids. 16 Fasting insulin levels are associated with adiposity. Differences in distribution of body fat particularly truncal subcutaneous fat in South Asians may account for the difference in fasting insulin. There is higher amount of fat and lower insulin sensitivity. $\underline{17}$ We used the cut off value of HOMA-IR at 2, specific for the South Asian population. This is based on the value of HOMA-IR at $75^{\text {th }}$ percentile of a Pakistani population.9 We used the cutoff value of fasting insulin specific for South Asian people $(10 \mathrm{mIU} / \mathrm{L})$ to define hyperinsulinemia. 9 The mean fasting insulin was above this cut-off value in both groups (insulin resistant and insulin sensitive) based on HOMA IR value. This is expected as the women are infertile polycystic ovary syndrome .

The prevalence of insulin resistance in our study is different from other studies. Studies on unselected population of polycystic ovary syndrome women have the prevalence of insulin resistance as $16 \%$ (Polish study, HOMA-IR >3.8), 3-30.7\% (Thai study, HOMA-IR >3.8). 11 The prevalence of insulin resistance is $76.9 \%$ in a study of Indian polycystic ovary syndrome women.12 The study measured insulin resistance by fasting glucose to insulin ratio $\leq 4.5$. The lower prevalence in our study may be because our group included younger women. A retrospective cross-sectional study of 538 Mexican infertile polycystic ovary syndrome women has the prevalence of insulin resistance $60.2 \%$. 13 They used the HOMA-IR cut-off value of 2.5 . The high prevalence may be due to the fact that $40.3 \%$ of their study population was overweight (BMI $\geq 25 \mathrm{~kg}$ / $\left.\mathrm{m}^{2}\right)$, and $44.3 \%$ was obese $\left(B M I \geq 30 \mathrm{~kg} / \mathrm{m}^{2}\right)$. The lack of uniformity in the methods of measurement of insulin resistance and the cut-off values of HOMA-IR is responsible for the difference in the prevalence of insulin resistance in different studies.

Women with polycystic ovary syndrome and overt oligomenorrhea have significantly more insulin resistance (HOMA-IR) than controls. $\underline{10}$ The study analyzed HOMA-IR variance among the groups of menstrual phenotypes. Free testosterone index is higher in women with insulin resistance.11 Insulin resistance leads to decreased hepatic generation of sex hormone binding globulin, so that free testosterone increases. We did not measure free testosterone, and the total testosterone did not show any difference. Studies on unselected population of women with polycystic ovary syndrome reveal that insulin resistance and hyperinsulinemia are in association with elevated triglyceride and low HDL cholesterol levels. $\underline{18}$ There is a study on 458 Korean women with polycystic ovary syndrome, who were divided into 4 groups by quartiles of HOMA-IR. Then the anthropometry, hormones, glucose metabolic and lipid profiles were compared between the groups. There were significant difference between groups in body mass index, weight height ratio, triglyceride, total cholesterol, LDL cholesterol and HDL cholesterol levels. 19 Polycystic ovary syndrome women with insulin resistance (HOMA IR >3.8) had significantly higher triglyceride level. 11 A study on 65 Indian polycystic ovary syndrome women in the reproductive age shows that insulin resistance is associated with dyslipidemia and this association is independent of obesity. $\underline{12}$ These studies include polycystic ovary syndrome women of older age, both fertile and infertile as well adolescents. Women with anovulatory polycystic ovary syndrome may have higher total cholesterol, triglycerides, LDL cholesterol and lower HDL cholesterol than women with ovulatory polycystic ovary syndrome.20 The women in our study were all infertile due to anovulation and we did not have ovulatory controls. Besides infertility, other causes behind the failure to find significant difference in lipid parameters between insulin resistant and insulin sensitive group may be age, genetic, ethnic, cultural or lifestyle differences influencing lipid profile.

Besides insulin resistance, hyperandrogenemia and obesity may have independent and interrelated effects on lipid profiles in women with polycystic ovary syndrome. $\underline{\underline{21}}$ High levels of triglyceride, free fatty acids and oxidized LDL in serum may be responsible for mitochondrial dysfunction and enhanced release of reactive oxygen species leading to accelerated follicular atresia and ovarian damage. .22 Statins, commonly used in polycystic ovary syndrome women to lower lipids, also reduce inflammation, oxidative stress and hyperandrogenism. $\underline{23}$ 
Fasting insulin level appears to be more relevant to the reproductive dysfunction in polycystic ovary syndrome than HOMA-IR which reflects reduced sensitivity of insulin suppe-ssion to exogenous or endogenous glucose. Hyperinsulinemia means that basal secretion of insulin from pancreatic beta cells is increased. The ovarian theca cells in polycystic ovary syndrome women are sensitive even to physiological levels of insulin. 4 Insulin acts via insulin receptors as well as IGF-1 receptors on theca cells of ovary to stimulate androgen production. Insulin also potentiates the stimulatory effect of luteinizing hormone on theca cells producing androgen. 5 Insulin's action of inhibiting hepatic SHBG production further contributes to hyperandro -genemia. Insulin resistance is prevalent in women with poly-cystic ovary syndrome both obese and lean. Hyperandrogene-mia aggravates insulin resistance. So, there is a self-propagating positive feedback loop of hyperinsulinemia and hyperandro -genemia, increasing over time. Paradoxically in women with polycystic ovary syndrome, the stimulatory effect of insulin on androgen production is persistent despite this apparent insulin resistance. 6 Insulin resistance occurs when peripheral tissues like liver, adipose tissues and muscle has less than normal insulin mediated response to glucose load. So, there is compensatory hyperinsulinemia. Fasting hyperinsulinemia may result from decreased hepatic clearance of insulin as well. 3 Dysglycemia and type II diabetes develop when pancreatic beta cells are incapable of insulin secretion sufficient to compensate for peripheral insulin resistance. $?$

According to our study, hyperinsulinemia defined by fasting insulin $\geq 10 \mathrm{IU} / \mathrm{L}$ has significant association with metabolic syndrome. Fasting insulin may be a better predictor of metabolic syndrome than HOMA-IR in infertile women with polycystic ovary syndrome. We should go beyond infertility management to counsel about the risk of diabetes mellitus and cardiovascular diseases in these women.

Analysis of variance between the four groups defined by quartiles of HOMA-IR in our infertile women with polycystic ovary syndrome resulted in significant difference in body mass index and WC between groups. Waist circumference and central obesity defined by waist circumference at or more than $80 \mathrm{~cm}$ predict insulin resistance as good as body mass index. While addressing insulin resistance by weight management, waist circumference and central obesity are as useful as body mass index for clinical evaluation of the progress. In fact waist circumference is easier to measure than body mass index. Of all the anthropometric measurements of obesity, waist circum-ference is most strongly associated with insulin sensitivity as measured by the gold standard test Euglycemic hyperinsulinemic clamp. Body fat mass has been measured by
CT (computerized tomographic scan) and DEXA (Dual-energy X-ray absorptiometry). $\underline{\underline{4}}$ Central obesity reflects both subcutaneous and visceral fat. Insulin resistance promotes differentiation of preadipocytes to adipocytes, particularly in abdominal area. Adipose tissue is a dynamic organ secreting adipokines, cytokines and hormones, contributing to the endocrine process that regulates reproduction in women with polycystic ovary syndrome. $\underline{25}$

Strength of the study is that it uses cut off levels specific for South Asian population for defining hyperinsulinemia and insulin resistance.

\section{Conclusion}

Insulin resistance defined by HOMA-IR cannot be predicted by most of the clinical, endocrine and metabolic parameters in infertile women with polycystic ovary syndrome except body mass index and waist circumference. Reducing body mass idex and waist circumference may improve insulin resistance in infertile women with polycystic ovary syndrome. Insulin resistance in these women with polycystic ovary syndrome is independent of lipid profiles. Hyperinsulinemia is significantly associated with metabolic syndrome.

\section{Funding Support}

Self-funded

\section{Ethical Issue}

The protocol for this study was approved by the Institutional Review Board of Bangabandhu Sheikh Mujib Medical University.

\section{Conflict of Interest}

Authors declare no conflict of interest

\section{References}

1. Rotterdam ESHRE/ASRM-sponsored PCOS consensus workshop group, Revised 2003 consensus on diagnostic criteria and long term health risks related to polycystic ovary syndrome (PCOS). Hum Reprod. 2004; 19: 41-47.

2. Dunaif $\mathrm{A}, \mathrm{Wu} \mathrm{X}$, Lee A, Diamanti-Kandarakis E. Defects in insulin receptor signaling in vivo in the polycystic ovary syndrome (PCOS). Am J Physiol Endocrinol Metab. 2001; 281: E392-E99.

3. Nawrocka-Rutkowska J, Ciecwiez S, Marciniak A, Brodowska A, Wisniewska B, Kotlega D. Insulin resistance assessment in patients with polycystic 
ovary syndrome using different diagnostic criteriaimpact of metformin treatment, Ann Agric Environ Med. 2013; 20: 528-32.

4. Nelson -Degrave VL, Wickenheisser JK, Hendricks KI, Asano T, Fujishiro M, Legro RS et al. Alterations in mitogen activated protein kinase kinase and extracellular regulated kinase signaling in theca cells contribute to excessive androgen production in polycystic ovary syndrome. Mol Endocrinol. 2005; 19: 379-90.

5. Wu XK, Zhou SY, Liu JX, Pollanen P, Sallinen K, Makinen M. Selective ovary resistance in insulin signaling in women with polycystic ovary syndrome. Fertil Steril. 2003; 80: 954-65.

6. Book CB, Dunaif A. Selective insulin resistance in the polycystic ovary syndrome. J Clin Endocrinol Metab. 1999; 84: 3110-16.

7. Legro RS. A fasting glucose to insulin ratio is a useful measure of insulin sensitivity in women with polycystic ovary syndrome. J Clin Endocrinol Metab. 1998; 83: 2694-98.

8. Wongwananuruk $\mathrm{T}$, Rattanachaiyanont $\mathrm{M}$ Leerasiri P, Indhavivadhana S, Techatrisak K, Surasak A et al. The usefulness of Homeostatic Measurement Assessment-Insulin Resistance (HOMA-IR) for detection of glucose intolerance in Thai women of reproductive age with polycystic ovary syndrome. Int J Endocrinol. 2012; 2012.

9. Hydrie MZI, Basit A, Fawwad A, Ahmedani MY, Shera MS, Hussain A. Detecting insulin resistance in Pakistani subjects by fasting blood samples. Open Diab J. 2012; 5: 20-24.

10. Brower M, Brennan K, Pall M, Azziz R. The severity of menstrual dysfunction as a predictor of insulin resistance in PCOS. J Clin Endocrinol Metab. 2013; 98: E1967-E71.

11. Pantasri T, Vutyavanich T, Shreshthaputra $O$, Srisupandit K, Piromlertamorn W. Metabolic syndrome and insulin resistance in Thai women with polycystic ovary syndrome. J Med Assoc Thai. 2010: 93: 406-12.

12. Kalra A, Nair S, Rai L. Association of obesity and insulin resistance with dyslipidemia in Indian women with polycystic ovary syndrome. Indian J Med Sci. 2006; 6: 445-53.

13. Reyes-Munoz E, Ortega-Gonzalez C, MartinezCruz N,Arche-Sanchez L, Estrada-Gutierrez G, Moran $\mathrm{C}$ et al. Association of obesity and overweight with the prevalence of insulin resistance, pre-diabetes and clinical-biochemical characteristics among infertile Mexican women with polycystic ovary syndrome: a cross-sectional study. BMJ Open. 2016; 2016.

14. Alberti KG, Eckel RH, Grundy SM, Zimmet PZ, Cleeman JI, Donato KA et al. Harmonizing the metabolic syndrome: A joint interim statement of the International Diabetes Federation Task Force on
Epidemiology and prevention; National Heart, Lung and Blood Institute; American Heart Association; World Heart Federation, International Atherosclerosis Society and International Association for the Study of Obesity. Circulation 2009; 120: 1640-45.

15. Trikudanathan S, Raji A, Chamarthi B, Seely EW, Simonson DC. Comparison of insulin sensitivity measures in South Asians. Metabolism 2013; 62: 1448-54.

16. Manjoo P, Dannenbaum D, Joseph L, Torrie J, Dasgupta K. Utility of current obesity thresholds in signaling diabetes risk in the James Bay Cree of EeyouIstchee. BMJ Open Diabetes Res Care. 2015; 2015.

17. Vikram NK, Misra A, Pandey RM, Luthra K, Bhatt SP. Distribution and cut-off points of fasting Insulin in Asian Indian adolescents and their association with metabolic syndrome. J Assoc Physicians India. 2009; 56: 949-56.

18. Baldani DP, Skrgatic L, Ougouag R. Polycystic ovary syndrome: Important underrecognized cardiometabolic risk factor in reproductive-age women. Int J Endocrinol. 2015; 2015.

19. Lee DE, Park SY, Park SY, Lee SR, Chung SW, Jeong K. Clinical and biochemical profiles according to Homeostasis Model Assessment-insulin Resistance (HOMA-IR) in Korean women with polycystic ovary syndrome. J Menopausal Med. 2014; 20: 104-10.

20. Rizzo M, Berneis K, Hersberger M, Pepe I, Di Fede G, Rini GB, et al. Milder forms of atherogenic dyslipidemia in ovulatory versus anovulatory polycystic ovary syndrome phenotype. Hum Reprod. 2009; 24: 2286-92.

21. Studen KB, Pfeifer M. Cardiometabolic risk in polycystic ovary syndrome. Endocr Connect. 2018; 7: R238-R51.

22. Schube U, Nowicki M, Jogschies P, Blumenauer V, Bechmann I, Serke H. Resveratrol and desferoxamine protect human OxLDL-treated granulosa cell subtypes from degeneration. J Clin Endocrinol Metab. 2014; 99: 229-39.

23. Liu Q, Xie Y, Qu L, Zhang M Mo Z. Dyslipidemia involvement in the development of polycystic ovary syndrome. Taiwan J Obstet Gynecol. 2019; $58: 447-53$.

24. Glintborg D, Petersen MH, Ravn P, Hermann AP, Andersen M. Comparison of regional fat mass measurement by whole body DXA scans and anthropometric measures to predict insulin resistance in women with polycystic ovary syndrome. Acta Obstet Gynecol Scand. 2016; 95: 1235-43.

25. Spritzer PM, Lecke SB, Satler F, Morsch DM. Adipose tissue dysfunction, adipokines, and lowgrade chronic inflammation in polycystic ovary syndrome. Reproduction 2015; 149: R219-R27. 